BMJ Open Diabetes

Research \& Care

\title{
A novel approach to glycemic control in type 2 diabetes mellitus, partial jejunal diversion: pre-clinical to clinical pathway
}

\author{
Martin Fried, ${ }^{1,2}$ Karin Dolezalova,,${ }^{1,2}$ Adam P Chambers, ${ }^{3}$ Elliott J Fegelman, ${ }^{4}$ \\ Robin Scamuffa, ${ }^{4}$ Michael L Schwiers, ${ }^{4}$ Jason R Waggoner, ${ }^{4}$ Martin Haluzik, ${ }^{5}$ \\ Randy J Seeley ${ }^{6}$
}

To cite: Fried M, Dolezalova K, Chambers AP, et al. A novel approach to glycemic control in type 2 diabetes mellitus, partial jejunal diversion: preclinical to clinical pathway. BMJ Open Diab Res Care 2017;5:e000431. doi:10.1136/ bmjdrc-2017-000431

Received 17 April 2017 Revised 20 July 2017 Accepted 26 July 2017

\section{(a) CrossMark}

${ }^{1}$ OB Klinika a.s, Prague, Czech Republic

${ }^{2} 1$ st Faculty of Medicine, Charles University, Prague, Czech Republic

${ }^{3}$ GLP-1 \& Obesity

Pharmacology, Novo Nordisk A/S, Malov, Denmark

${ }^{4}$ Ethicon, Inc, Cincinnati, OH, USA

${ }^{5}$ Institute for Clinical and Experimental Medicine, Centre for Experimental Medicine, Diabetes Centre, Prague, Czech Republic

${ }^{6}$ Department of Surgery, Internal Medicine and Nutritional Science, University of Michigan, Ann Arbor, MI, USA

Correspondence to Dr Jason R Waggoner; jwaggon1@its.jnj.com

\section{ABSTRACT}

Objective To explore partial jejunal diversion (PJD) via a side-to-side jejuno-jejunostomy for improved glycemic control in type 2 diabetes mellitus (T2DM). PJD is an anatomy-sparing, technically simple surgery in comparison to the predominate metabolic procedures, Roux-en-Y gastric bypass (RYGB) and sleeve gastrectomy (SG). Positive results in a rodent model prompted a human proof-of-concept study.

Research design and methods Pre-clinically, 71 rats were studied in a model of metabolic dysfunction induced by a high-fat diet; 33 animals undergoing one of two lengths of PJD were compared with 18 undergoing sham, 10 RYGB and 10 jejuno-ileal bypass. Clinically, 15 adult subjects with treated but inadequately controlled T2DM (hemoglobin A1c ( $\mathrm{HbA1C}$ ) of $8.0 \%-11.0 \%$ ), body mass index of $27.0-40.0 \mathrm{~kg} / \mathrm{m}^{2}$, and $C$ peptide $\geq 3 \mathrm{ng} / \mathrm{mL}$ were studied. Follow-up was at 2 weeks, and 3, 6, 9, and 12 months post-PJD.

Results Pre-clinically, positive impacts with PJD on glucose homeostasis, cholesterol, and body composition versus sham control were demonstrated. Clinically, PJD was performed successfully without serious complications. Twelve months post-surgery, the mean (SD) reduction from baseline in $\mathrm{HbA1c}$ was $2.3 \%(1.3)(\mathrm{p}<0.01)$.

Conclusions PJD may provide an anatomy sparing, low-risk, intervention for poorly controlled T2DM without significant alteration of the patient's lifestyle. The proofof-concept study is limited by a small sample size and advanced disease, with $80 \%$ of participants on insulin and a mean time since diagnosis of over 10 years. Further study is warranted.

Trial registration number NCT02283632; Pre-results.

\section{INTRODUCTION}

WHO reports that 422 million adults have diabetes, the majority of whom are affected by type 2 diabetes mellitus (T2DM). ${ }^{1}$ T2DM was the sixth leading cause of death in $2015 .^{2}$ Bariatric metabolic surgeries (Roux-en-Y gastric bypass (RYGB) and sleeve gastrectomy (SG) ) have emerged as the most effective interventions for controlling T2DM in the obese. $^{3}$ The Second Diabetes Surgery Summit

\section{Significance of this study}

What is already known about this subject?

- Surgical procedures such as Roux-en-Y gastric bypass and sleeve gastrectomy induce sustained improved glycemic control in patients with type 2 diabetes mellitus (T2DM) by weight loss-independent and weight loss-dependent mechanisms. One mechanistic factor involved is the introduction of nutrients more quickly and distally in the small intestine.

What are the new findings?

- A human proof-of-concept study was successfully conducted in patients with T2DM utilizing a sideto-side jejuno-jejunostomy for partial diversion of nutrients more distally. In this small cohort of obese subjects with inadequately controlled T2DM of long duration, substantial, clinically relevant improvements in glycemic and weight control were observed.

How might these results change the focus of research or clinical practice?

- Partial jejunal diversion may provide an anatomy sparing, low-risk, potentially reversible, metabolic procedure for patients with poorly controlled T2DM, which does not impose significant alterations in lifestyle.

(DSS-II) recently concluded that there is sufficient clinical and mechanistic evidence to support inclusion of metabolic surgery among antidiabetic interventions for T2DM and obesity. ${ }^{4}$ However, not all patients are receptive to anatomy altering surgery and lifestyle changing procedures, and more than a third of the world's diabetic population is not obese.

Importantly, more than half the diabetic population is not under adequate glycemic control with current therapies. ${ }^{5}$ Metabolic studies indicate that introduction of 
nutrients more quickly and distally in the small intestine can improve glucose homeostasis. A recent study ${ }^{6}$ indicates that bariatric surgery can result in complete or partial T2DM remission in a majority of patients for at least 5 years and provides the impetus to develop less invasive metabolic procedures. ${ }^{7}$ The jejuno-ileal bypass (JIB), ${ }^{89}$ the duodenal-jejunal bypass (DJB), ${ }^{7}$ DJB with SG, the single-anastomosis duodenoileal bypass with SG (SADI-S) ${ }^{10}$ and the SG plus side-to-side jejuno-ileal anastomosis ${ }^{11}$ procedures have shown improvements in glucose homeostasis but have had limited adoption due to a combination of surgical complications and negative impact on lifestyle.

A rodent model with a side-to-side jejuno-jejunostomy is reported here which indicates that a partial diversion of stomach contents in the small intestine improves glycemic control with less weight loss than current bariatric metabolic surgeries. By utilizing a side-to-side jejuno-jejunostomy, complications such as severe diarrhea seen with the JIB procedure may be avoided. Partial jejunal diversion (PJD) entails creating a side-to-side anastomosis that allows a portion of nutrients to bypass the intact loop of bowel while the remaining portion of nutrients follows the common path of intestinal transit. This procedure intentionally eliminates the blind limb associated with JIB. Given the demonstrated improvements in glucose tolerance in a pre-clinical model, a proof-of-concept study was undertaken in patients with poorly controlled T2DM with a body mass index (BMI) of $27-40 \mathrm{~kg} / \mathrm{m}^{2}$.

\section{RESEARCH DESIGN AND METHODS}

Rat model of diet-induced obesity and metabolic impairment Male Long-Evans rats ( $\mathrm{n}=71$ ) (250-300 g; Harlan Laboratories, Indianapolis, Indiana, USA) were individually housed and maintained on a 12/12 hour light/dark cycle (lights off at $18: 00$ hours) at $25^{\circ} \mathrm{C}$ and $50 \%-60 \%$ humidity. All procedures for animal use were approved by the University of Cincinnati Institutional Animal Care and Use Committee. Before surgery, rats were given ad libitum access to water and a high-fat diet $(4.54 \mathrm{kcal} / \mathrm{g} ; 41 \%$ fat; Research Diets, New Brunswick, New Jersey, USA) previously documented to produce metabolic impairments. ${ }^{12}$ After 8 weeks on the high-fat diet, rats were assigned to one of five surgical groups (sham, JIB, $30 \mathrm{~cm}$ PJD, $42 \mathrm{~cm}$ PJD or RYGB) that were matched for fat tissue mass. At 3 days pre-operatively, the high-fat diet was temporarily replaced with Ensure Plus liquid diet (1.41 kcal/g; 29\% fat) (Abbott Nutrition, Columbus, Ohio, USA) for 7 days after which the rats were returned to the same high-fat diet as before. Subcutaneous injections of Metacam $(0.25 \mathrm{mg} / 100 \mathrm{~g}$ body weight (BW) once daily for 4 days $)$, gentamicin $(0.8 \mathrm{mg} / 100 \mathrm{~g} \mathrm{BW}$ on the day of surgery), Buprenex (0.3 mL $2 \mathrm{X}$ per day for 5 days), and warm saline ( 10 and $5 \mathrm{~mL} 2 \mathrm{X}$ per day for days $0-3$ and $4-5$, respectively) were given to all post-operative rats. On the day of surgery, rats were anesthetized under isoflurane and pre-treated with analgesics and gentamicin as above.

\section{Rodent models}

For the sham operation, a laparotomy was performed to gain access to the abdominal cavity, and a section of jejunum was isolated and cut $30 \mathrm{~cm}$ beyond the ligament of Treitz. The two halves were then anastomosed end-to-end using a running stitch with 7-0 Vicryl absorbable suture (Ethicon, Somerville, New Jersey, USA). The laparotomy was closed in layers. For the $30 \mathrm{~cm}$ PJD, an anti-mesenteric incision was made $30 \mathrm{~cm}$ beyond the ligament of Treitz and another $30 \mathrm{~cm}$ from the ileocecal junction. A running stitch with 8-0 suture was used to join the two sections of bowel with reinforcements placed at the mid-point of each semi-circular section with an interrupted stitch. The resulting loop was roughly $30 \mathrm{~cm}$ long. The $42 \mathrm{~cm}$ PJD was identical except that the distal incision was made $18 \mathrm{~cm}$ from the ileocecal junction. For JIB an anti-mesenteric incision was made $30 \mathrm{~cm}$ from the ileocecal junction. The duodenum was transected $10 \mathrm{~cm}$ beyond the pylorus and the distal jejunal remnant was closed. The two open sections of bowel were then connected using a running stitch with reinforcements at each mid-point. To perform RYGB, the jejunum was transected $30 \mathrm{~cm}$ beyond the ligament of Treitz. An anti-mesenteric incision was made $10 \mathrm{~cm}$ distal to the transected bowel and connected to the afferent limb with a running absorbable suture. The stomach was isolated and the fundus was excised by making a vertical cut along the edge of the corpus with an ETS Articulating Linear Cutter (Ethicon, Cincinnati, Ohio, USA). A second staple line was placed across the waist of the stomach, creating a gastric pouch that was $10 \%$ the size of the original stomach. The distal remnant was returned to the peritoneal cavity and an incision was made on the gastric pouch that spared the vascular architecture. The efferent limb of the transected jejunum was then connected to the gastric pouch with a running 8-0 Prolene non-absorbable suture (Ethicon, Somerville). All incisions used to create anastomoses were $8 \mathrm{~mm}$ long and in all cases the abdominal wall was closed in layers using a running stitch and a running subcuticular stitch.

\section{Rodent food intake and fat absorption}

A pilot study was performed in a separate cohort of rats $(n=22 / 71)$ from those used in the meal tolerance test to assess the effect of PJD on food intake and fat absorption. Food intake was monitored weekly after surgery in sham $(\mathrm{n}=8), 30 \mathrm{~cm}$ PJD $(\mathrm{n}=7)$, or $42 \mathrm{~cm}$ PJD $(\mathrm{n}=7)$ treatment groups and fat absorption was assessed after 4 weeks using the Behenate method, as described previously. ${ }^{13}$ Briefly, the high-fat diet was removed and rats were temporarily placed on a diet containing $5 \%$ sucrose polybehenate (behenic acid). After 24 hours of acclimation to the diet, cages were changed and fecal pellets were collected after another 24 hours. Fecal samples of about $10 \mathrm{mg}$ were collected and fecal lipid content was assayed by gas chromatography of fatty acid methyl esters. Fat absorption was calculated from the ratio of behenic acid to other fatty acids in the diet and feces. ${ }^{13}$ 
Rodent mixed-meal tolerance test

Blood glucose, glucagon-like peptide-1 (GLP-1), and insulin response to a mixed-meal tolerance test (MMTT) were assessed in rats that underwent sham $(n=10), 30 \mathrm{~cm}$ PJD $(n=8), 42 \mathrm{~cm}$ PJD $(n=11)$, JIB $(n=10)$, or RYGB $(\mathrm{n}=9)$ procedures. Approximately 5 weeks after surgery, 5 -hour fasted rats were gavaged with $2.8 \mathrm{~mL}$ Ensure Plus Liquid diet. Blood samples $(5 \mu \mathrm{L})$ for glucose were collected at $0,15,30,45,60,120 \mathrm{~min}$ post-gavage, and analyzed by a hand-held glucometer to quantify glucose concentrations. An additional $180 \mu \mathrm{L}$ aliquot of blood was collected into tubes containing $20 \mathrm{~L}$ antiproteolytic cocktail (4.65 g EDTA, $92 \mathrm{mg}$ aprotinin, $40000 \mathrm{U}$ heparin in $50 \mathrm{~mL}$ saline) at baseline and $15 \mathrm{~min}$ after gavage to assess post-prandial plasma GLP-1 and insulin release. The 15 min time point was chosen to coincide with peak plasma insulin and GLP-1 levels in sham and RYGB rats observed previously by our group. ${ }^{14}$ We do not know if this time point coincides with peak levels in PJD rats. Samples were centrifuged $(20 \mathrm{~min}$ at $9000 \mathrm{rpm})$ and plasma was collected and immediately frozen until analysis. GLP-1 was analyzed by an electrochemiluminescence assay (Meso Scale Discovery, Gaithersburg, Maryland, USA) and plasma insulin was measured using a rat insulin ELISA (Crystal Chem, Downers Grove, Illinois, USA).

\section{Rodent body composition}

Lean and fat tissue mass were assessed using an EchoMRI analyzer (Houston, Texas, USA) 1 week prior to surgery and again after 4 weeks in sham $(n=10), 30 \mathrm{~cm}$ PJD $(n=8)$, $42 \mathrm{~cm}$ PJD $(n=10)$, JIB $(n=10)$, and RYGB $(n=9)$ rats. A rat in the $42 \mathrm{~cm}$ PJD group died prior to the final body weight measurement on day 56 and was excluded from body composition and liver triglyceride (TG) analyses.

\section{Rodent liver TG}

On day 56, the same rats used in the MMTT were sacrificed using $\mathrm{CO}_{2}$ asphyxiation. Livers were flash frozen in isopentane, and lipid from $50 \mathrm{mg}$ of tissue was extracted in 2:1 chloroform/methanol. TG content was measured via colorimetric assays using Infinity Reagents (Thermo Fisher Scientific, Waltham, Massachusetts, USA).

\section{Clinical study design, recruitment, and enrollment of participants}

Fifteen patients underwent PJD between November 2014 and May 2015 at the OB Clinic, Prague, Czech Republic. The study was approved by the Ethics Committee of the OB Clinic and informed consent was obtained from all study patients. The study was conducted in accordance with the Declaration of Helsinki and was registered at ClinicalTrials.gov supported by the United States National Institutes of Health as NCT02283632.

Key inclusion criteria included hemoglobin A1c (HbAlc) of $8.0 \%-11.0 \%$, BMI $27.0-39.9 \mathrm{~kg} / \mathrm{m}^{2}$, age 20-60 years, $\mathrm{C}$ peptide $\geq 3 \mathrm{ng} / \mathrm{mL}$, and presence of at least one other cardiovascular risk factor: hypertension (systolic blood pressure (BP) $\geq 140 \mathrm{~mm} \mathrm{Hg}$ and/or diastolic $\mathrm{BP} \geq 90 \mathrm{~mm} \mathrm{Hg}$ ) or anti-hypertensive medication use; dyslipidemia (high-density lipoprotein cholesterol (HDL-C) $<40 \mathrm{mg} / \mathrm{mL}$ (men) or $<50 \mathrm{mg} / \mathrm{mL}$ (women); low-density lipoprotein cholesterol (LDL-C) $>100 \mathrm{mg}$ / $\mathrm{mL}$; or $\mathrm{TG} \geq 150 \mathrm{mg} / \mathrm{dL}$ ) or dyslipidemia medication use.

Key exclusion criteria included any previous major gastrointestinal (GI) resection, type 1 diabetes mellitus, latent autoimmune diabetes of adulthood, screening laboratory test abnormalities (alanine aminotransferase (ALT) and/or aspartate aminotransferase (AST) levels $\geq 4$ times upper limit of normal (ULN); blood creatinine level $\geq 1.5$ times ULN; or blood urea nitrogen (BUN) level $\geq 1.5$ times ULN), or prescription or over-the-counter medications or supplements with a primary indication known to cause or assist in weight reduction.

\section{Procedures}

All patients had a physical examination conducted, and fasting blood laboratory samples, medical history, concomitant medication usage, vital signs and anthropometric data collected prior to PJD. Follow-up visits were conducted at 2 weeks, 3, 6, 9, and 12 months after the procedure. Per local medical practice, patients were hospitalized and discharged only after normal bowel movement. For each visit, vital signs, anthropometric data, fasting blood laboratory data, and concomitant medication usage were recorded. Oral glucose tolerance testing (OGTT) was performed at the baseline visit and at 3 and 12 months post-procedure. A CT enterography (CTE) scan was conducted to assess anastomotic patency approximately 9-12 months after PJD. The scans were read by an independent radiologist to subjectively assess percent of nutrient diversion.

\section{Surgical technique}

Patients underwent general anesthesia, and optimal laparoscopic access and pneumoperitoneum were established. Four trocars (two $5 \mathrm{~mm}$ and two $10 / 12 \mathrm{~mm}$ ) were placed. One $10 / 12 \mathrm{~mm}$ was introduced in the mid-hypogastric region and the second $10 / 12 \mathrm{~mm}$ mid-distance between the right iliac spine and umbilicus. One $5 \mathrm{~mm}$ trocar was placed on the patient's left, opposite to the $10 / 12 \mathrm{~mm}$ in the left iliac region and the second $5 \mathrm{~mm}$ trocar was placed in the patient's left flank on the umbilical level. The omentum was retracted cephalad while simultaneously exposing the ligament of Treitz. The jejunum was measured $100 \mathrm{~cm}$ from the ligament of Treitz and marked with a suture. A length of $250 \mathrm{~cm}$ of ileum was measured from the ileocecal junction. At the site, the jejunal limb was approximated and aligned with the ileum in an isoperistaltic manner. The anti-mesenteric borders of the jejunal and ileal limbs were opened using an advanced energy bipolar device. Two enterotomies were created and a $60 \mathrm{~mm}$ long linear stapler was used to create a side-to-side anastomosis. The lumen was inspected for bleeding and the enterotomy was hand sutured closed in two layers. The mesenteric defect along the anastomosis was closed by running suture. 
Finally, the abdomen was deflated and the port sites were closed.

\section{Outcomes}

All subjects were assessed for HbAlc at 2 weeks, and 3, 6, 9, and 12 months post-procedure and change from baseline in HbAlc was calculated. Other pre-specified outcome measurements included safety, change from baseline in glucose homeostasis (blood glucose area under curve (AUC) values at each visit were calculated using the linear trapezoidal rule based on the fasting value prior to the glucose tolerance test and the subsequent values collected at $30 \mathrm{~min}$, and $1,2,3,4$, and 5 hours after the glucose tolerance test), whole-body insulin sensitivity (assessed from the OGTT data using the Matsuda Index ${ }^{15}$ ), $\beta$-cell function (assessed using the ratio of AUC C peptide/AUCglucose calculated from the OGTT data ${ }^{16}$ ), lipid levels, liver and renal function, and usage of anti-hyperglycemic, anti-hypertensive, and dyslipidemia medications.

\section{Statistical analysis}

Rodent data were analyzed via one-way independent analysis of variance (ANOVA) or two-way ANOVA with time as a repeated measure, followed by Bonferroni's multiple comparisons tests, or, unpaired t-tests where indicated. Data are expressed as mean \pm SEM.

For statistical analyses of all clinical data, SAS, V.9.3 (Cary, North Carolina, USA) was used. Summary statistics including $\mathrm{n}$, mean, SD, median, minimum, and maximum were provided for the observed values at the given time point as well as the change from baseline at that time point. Additionally, 95\% CIs were estimated for the change from baseline and the mean change from baseline was tested using the one-sample t-test or the Wilcoxon signed-rank test as appropriate. A significance level of 0.05 was used to determine statistical significance. For all analyses, baseline was defined as the last measurement obtained prior to PJD being performed.

\section{RESULTS}

\section{Pre-clinical results}

During the first 2 weeks post-operatively, food intake was significantly reduced in rats undergoing $30 \mathrm{~cm}$ PJD $(731 \pm 40 \mathrm{~g}, \mathrm{n}=8)$ and $42 \mathrm{~cm}$ PJD $(650 \pm 77 \mathrm{~g}, \mathrm{n}=7)$ relative to sham-operated rats $(1054 \pm 73 \mathrm{~g}, \mathrm{n}=8)$, after which differences in weekly food consumption were non-significant among groups (data not shown). Fat absorption, expressed as a percent of dietary content absorbed, was slightly reduced in rats that underwent $42 \mathrm{~cm}$ PJD $(75 \% \pm 3.3 \%, \mathrm{n}=7)$ relative to sham-operated rats $(83 \% \pm 1.6 \%, \mathrm{n}=8 ; \mathrm{p}=0.03)$, whereas differences in fat absorption between sham and $30 \mathrm{~cm}$ PJD $(77 \% \pm 2.8 \%$, $\mathrm{n}=8$ ) rats were non-significant.

In a second cohort of rats, blood glucose, plasma GLP-1, and plasma insulin levels were assessed during a MMTT performed approximately 5 weeks after surgery. Figure 1B shows that both $30 \mathrm{~cm}$ (at $15 \mathrm{~min}$ ) and $42 \mathrm{~cm}$ (at $120 \mathrm{~min}$ ) versions of PJD resulted in significantly lower blood glucose excursions than sham during the MMTT. As expected, plasma GLP-1 levels (Figure 1C) were elevated after RYGB and JIB 15 min post-MMTT relative to sham-operated rats, and to a greater extent than after PJD. However, this may have been different at later time points. Interestingly, both fasting and post-challenge plasma insulin levels (Figure 1D) were significantly lower after $30 \mathrm{~cm}$ PJD than sham, implying greater insulin sensitivity as body weight, body composition, and liver TG levels were similar among these groups (Figure $1 \mathrm{~A}, \mathrm{E}-\mathrm{G}$ ). RYGB and JIB resulted in greater blood glucose lowering than PJD but also evoked significantly more weight loss.

\section{Clinical results}

\section{Patient characteristics}

Baseline characteristics are shown in table 1 for the 15 subjects undergoing PJD.

\section{Procedure}

PJD was successfully completed in all 15 patients. The mean procedure duration was $104.5 \mathrm{~min}$ (range: $70-140 \mathrm{~min}$ ). The average length of hospital stay was 3.3 nights (range: 2-7 nights). All patients were discharged uneventfully and returned to normal diet immediately. There were no readmissions after discharge. CTE scans showed that the anastomosis was patent at 9-12 months after the procedure. The mean oral jejunum to oral ileum flow was estimated to be $57 \%-43 \%$, respectively.

\section{HbA1c and glucose homeostasis}

Mean HbA1c (figure 2A and table 2) and fasting blood glucose (table 2 ) were significantly lower by 2 weeks after PJD, and continued to decrease over 12 months. Eight of 15 patients $(53.3 \%)$ had a $>2 \%$ absolute reduction and 11 of 15 patients $(73.3 \%)$ had a $>1.5 \%$ absolute reduction in HbA1c 12 months post-PJD, and 7 patients (46.7\%) had achieved a HbA1c $<7.0 \%$. Compared with baseline at 12 months post-PJD, OGTT showed a significant decrease in blood glucose AUC, a significant increase in $\beta$-cell function, a significant increase in GLP-1 AUC, and an increase in whole-body insulin sensitivity.

\section{Weight change}

Significant weight loss was first observed 2 weeks after PJD and continued over 12 months (figure 2B and table 2). Total weight loss varied from 2 to $21 \mathrm{~kg}$ with a mean of $10.8 \mathrm{~kg}$ (corresponding to a $10.3 \%$ reduction from baseline weight). Ten of 15 patients $(66.7 \%)$ lost $\geq 10 \%$ of their body weight and 13 of 15 patients $(86.7 \%)$ lost $\geq 5 \%$. Concurrent reductions were also observed in BMI and waist circumference.

\section{Other cardiometabolic outcomes}

Mean fasting insulin decreased significantly in conjunction with mean LDL-C and TG at 12 months post-procedure and there were no significant changes in mean HDL-C (table 2). Mean systolic BP decreased significantly at 12 months post-procedure, whereas diastolic BP remained 
A.

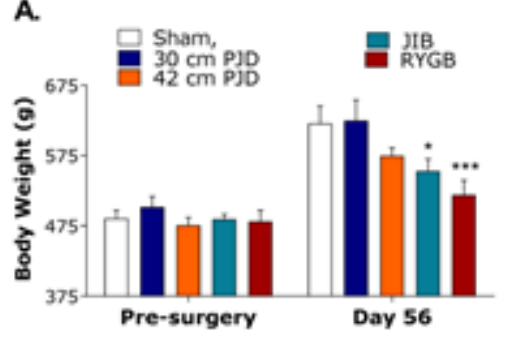

c.

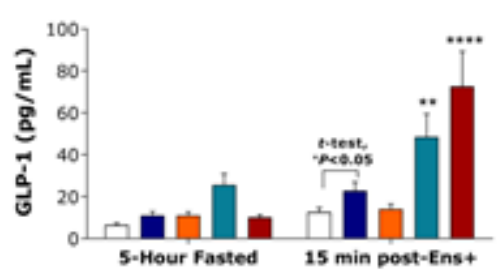

Lean Mass

E.

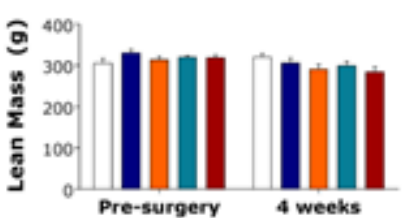

B.

F.

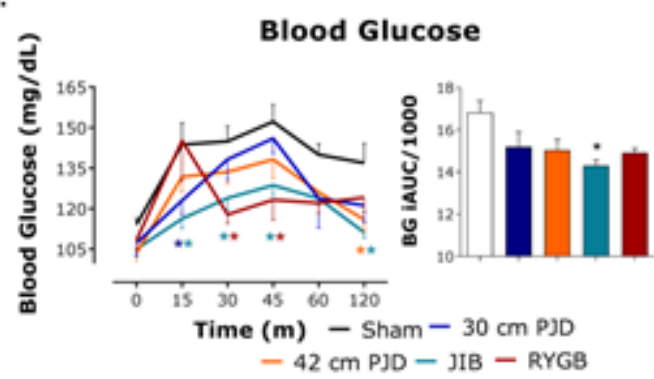

D.

Plasma Insulin

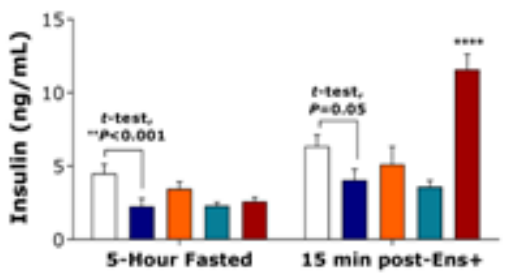

Fat Mass

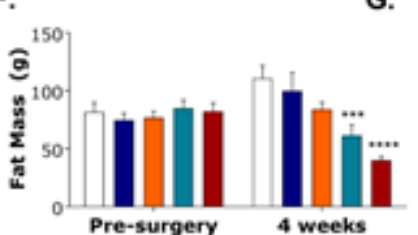

Liver Triglycerides

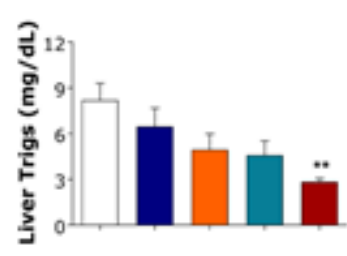

Figure 1 Body weight and oral glucose tolerance in diet-induced obese rats at 5 weeks post-surgery. Diet-induced obese rats underwent a sham $(n=10)$ operation, $30 \mathrm{~cm}$ partial jejunal diversion (PJD) $(n=8), 42 \mathrm{~cm}$ PJD ( $n=10)$, jejuno-ileal bypass (JIB) $(n=10)$, or Roux-en-Y gastric bypass (RYGB) ( $n=9)$ surgery. (A) Body weight (g); (B) blood glucose in response to a 2-hour mixed meal tolerance test (MMTT, n=7-11 per group); (C) plasma GLP-1 (pg/mL, 8-10 per group); (D) plasma insulin (ng/mL, $\mathrm{n}=8-10$ ); (E) 4-week lean mass ( $\mathrm{g}, \mathrm{n}=8-10$ per group); $\mathrm{F}$ ) 4-week fat mass ( $\mathrm{g}, \mathrm{n}=8-10$ per group); (G) liver triglycerides (mg/ $\mathrm{dL}$ ). All data presented as mean \pm SE. Data were analyzed via one-way independent measures analysis of variance (ANOVA) or two-way ANOVA with time as a repeated measure, followed by Bonferroni multiple comparisons test where appropriate. ${ }^{*} \mathrm{p}<0.05,{ }^{* *} \mathrm{p}<0.01,{ }^{* * *} \mathrm{p}<0.001,{ }^{* * *} \mathrm{p}<0.0001$, unpaired t-tests were performed where indicated.

essentially unchanged over the 12-month post-procedure period (table 2). All liver function values (except for serum albumin) decreased significantly at 12 months post-procedure (table 2). For renal function, creatinine remained similar to baseline values, but BUN increased significantly at 12 months post-procedure (table 2). Vitamin B-12 levels decreased significantly at 12 months post-procedure (table 2).

Pre-PJD, 14 patients were taking at least one anti-hyperglycemic agent (AHA) of whom 12 were receiving insulin; one patient had stopped all anti-hyperglycemic medication before entry into the study. Twelve months after PJD, 13 patients still required at least one AHA of whom 8 were receiving insulin, although 4 patients decreased the number of AHAs they received. Among the 12 patients requiring insulin at the start of the study, 4 stopped insulin treatment (baseline dose ranged from 30 to 80 units); 4 continued on insulin with an average reduction in their approximate daily dose of $54 \%$ (mean reduction of 55 units with range from -30 to -84 units); and another 4 maintained the same approximate daily dose of insulin (unit change of no more than 10 units). There were no major changes in number of patients receiving anti-hypertensive and dyslipidemia medications at study end (table 3).

\section{Clinical adverse events}

All adverse events (AEs) regardless of type, severity, or relationship to the procedure were recorded for the duration of the study. There were 36 procedure-related AEs recorded in 13 subjects (86.7\%) and one was considered severe, 11 were considered moderate, and 24 were considered mild. These procedure-related AEs were also classified for clinical relevance per the Clavien-Dindo surgical complications scale. ${ }^{17}$ There were 32 AEs at the lowest grade (grade I), 4 were grade II, and none reached grades III-V. No event resulted in surgical intervention. Fifteen events required no intervention; 2 resolved with diet; 19 resolved with medication including one episode of lower extremity 


\begin{tabular}{|c|c|}
\hline Characteristic & $\begin{array}{l}\text { Mean (SD) or Percent (n) } \\
(n=15)\end{array}$ \\
\hline Age (years) & $52.7(6.11)$ \\
\hline \multicolumn{2}{|l|}{ Sex } \\
\hline Female & $46.7 \%(7)$ \\
\hline Male & $53.3 \%(8)$ \\
\hline \multicolumn{2}{|l|}{ Race } \\
\hline White & $100.0 \%(15)$ \\
\hline \multicolumn{2}{|l|}{ Ethnicity } \\
\hline Not Hispanic or Latino & $100.0 \%(15)$ \\
\hline BMI $\left(\mathrm{kg} / \mathrm{m}^{2}\right)$ & 34.1 (3.51) Range: 27.4-39.8 \\
\hline Weight (kg) & $\begin{array}{l}104.3 \text { (17.34) Range: } 80.0- \\
138.0\end{array}$ \\
\hline Waist circumference (cm) & $121.5(16.19)$ \\
\hline $\begin{array}{l}\text { Years since diagnosis of } \\
\text { diabetes }\end{array}$ & 10.9 (5.95) Range: 4-26 \\
\hline
\end{tabular}

BMI, body mass index.

edema (considered severe) that resolved during a 2-day hospitalization.

Among the 13 subjects experiencing a procedure-related $\mathrm{AE}$, the most common AEs were diarrhea in 7 subjects $(46.7 \%)$, flatulence in 4 subjects $(26.7 \%)$, and pain in 5 subjects $(33.3 \%)$. Diarrhea dissipated between 2 and 10 days after onset and was considered mild to moderate. One episode of dehydration, not considered procedure-related, prompted hospitalization 3 months after the procedure, but was not associated with diarrhea and resolved without sequelae.

\section{CONCLUSIONS}

Based on the improvements in oral glucose tolerance in a rodent model of diet-induced obesity and metabolic impairment, PJD demonstrated potential as a surgical

A

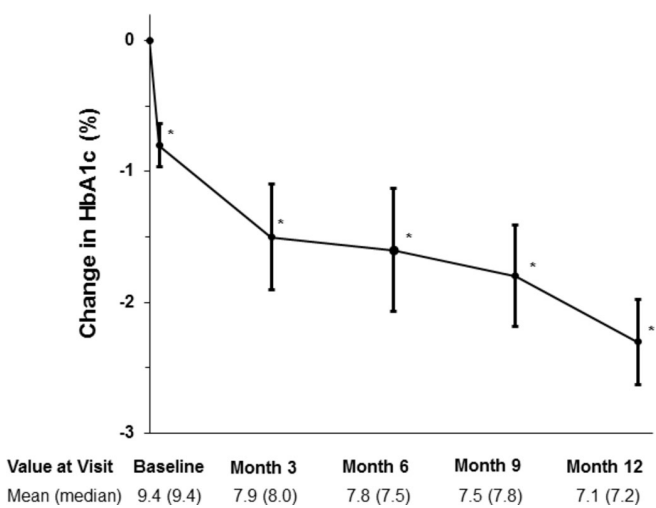

approach to treat T2DM. These data show that PJD is mildly or non-malabsorptive depending on distance of the intestinal loop along the GI tract. Overall, differences among 30 and $42 \mathrm{~cm}$ versions of PJD in the pre-clinical studies were subtle. There was a trend for the $42 \mathrm{~cm}$ PJD version of treated rats to weigh less and fat absorption was reduced by approximately $10 \%$ in this group relative to sham-treated rats, whereas fat absorption was unaffected in $30 \mathrm{~cm}$ PJD rats. In comparison, we previously showed that RYGB surgery reduces fat absorption by roughly $30 \%$ in this model. ${ }^{18}$ Taken together, these data show that PJD has the potential to benefit oral glucose handling without causing pronounced or drastic significant malabsorption or changes in body weight in a pre-clinical rat model. We do not yet know if, or, how these findings will translate to PJD in humans.

Clinically, PJD was executed as anticipated without incident, and was generally well-tolerated by the patients. Given historical experience with serious complications after JIB, ${ }^{8}{ }^{9} \mathrm{PJD}$ was designed with a partial diversion to avert the complications associated with a blind loop, full bypass. AEs were scrupulously recorded and rigorously addressed in this proof-of-concept study, resulting in an observed procedure-related $\mathrm{AE}$ rate of $86.7 \%$. However, classification of these events by their clinical relevance using the Clavien-Dindo scale indicated a favorable safety profile with all events at the grade I or II level and none at grades III-V. A recent evaluation ${ }^{19}$ of patients with diabetes undergoing an RYGB using a composite of complications (considered grade III or higher on the Clavien-Dindo scale) reported a 3.4\% complication rate. This work observed none of these complications, although it is recognized that this initial cohort is too small to observe events with a low incidence, thus warranting further observation in a larger population.

Even in this small cohort of predominantly obese subjects with advanced T2DM, substantial, clinically relevant improvements in glycemic and weight control were observed with attendant reduction in AHA usage. Based

B

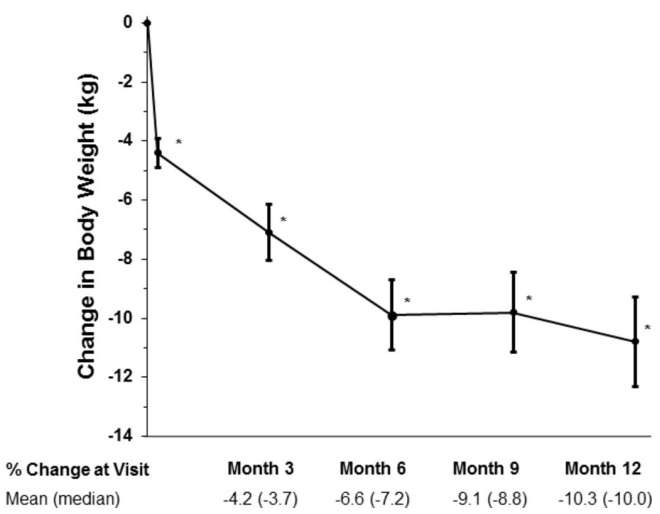

Figure 2 Key clinical efficacy outcomes for $(A)$ hemoglobin $A 1 c(H b A 1 c),(B)$ weight change. Data points are mean change from baseline absolute $\mathrm{HbA1c}(\mathrm{A})$ or mean change from baseline absolute body weight (B) and error bars represent SEM for $n=15$ patients. Data below the charts are absolute mean and median $\mathrm{HbA1c}(\mathrm{A})$ or mean and median $\%$ body weight reduction $\left({ }^{*} \mathrm{p}<0.05\right)$ compared with baseline values. 


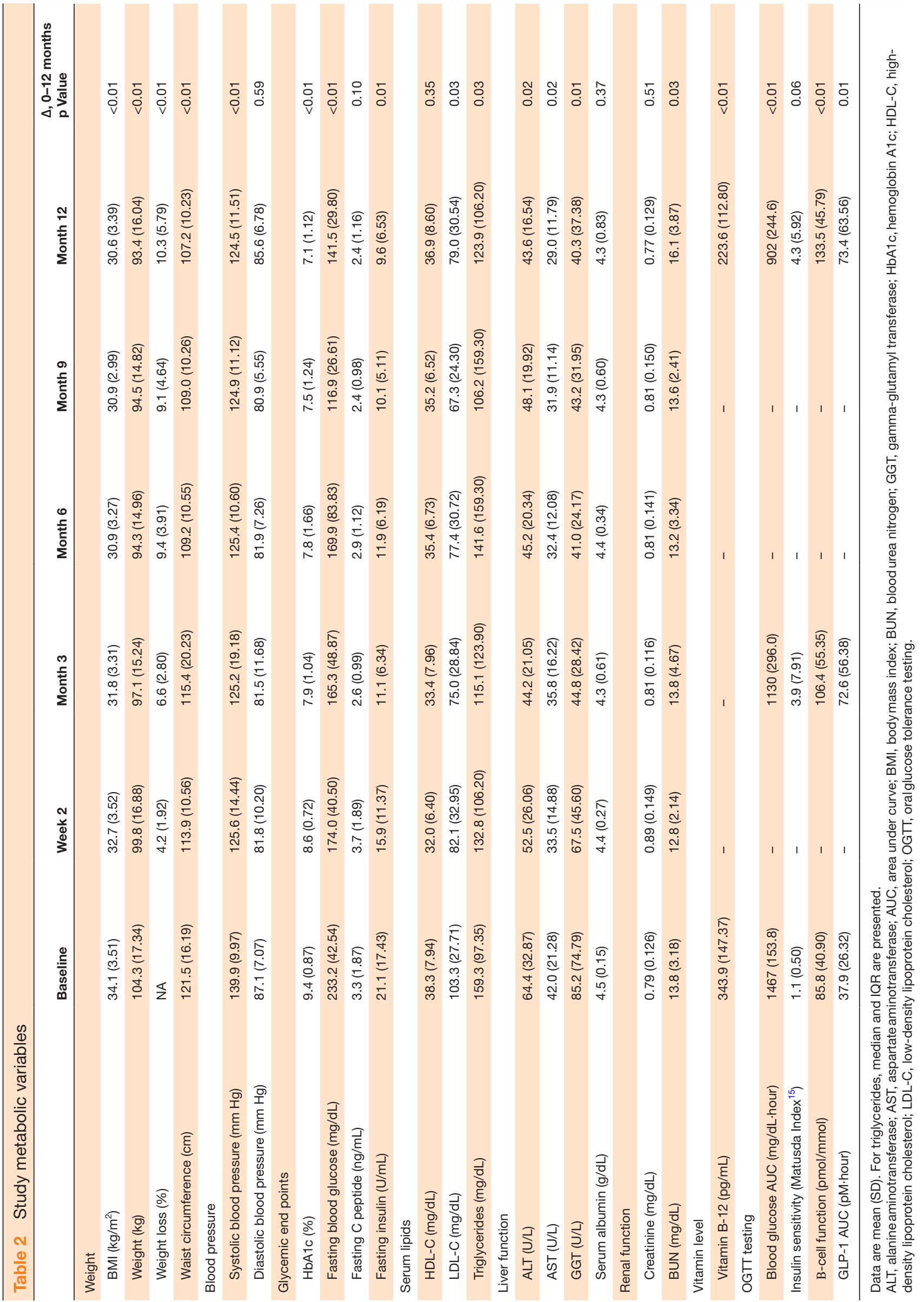




\begin{tabular}{|c|c|c|}
\hline & Baseline & Month 12 \\
\hline \multicolumn{3}{|l|}{ Concomitant medicines } \\
\hline At least $1 \mathrm{AHA}$ & $93.3 \%(14)$ & $86.7 \%(13)$ \\
\hline Insulin & $80.0 \%(12)$ & $53.3 \%(8)$ \\
\hline Other AHAs & $93.3 \%(14)$ & $86.7 \%(13)$ \\
\hline Anti-hypertensive agents & $73.3 \%(11)$ & $80.0 \%(12)$ \\
\hline Dyslipidemia agents & $66.7 \%(10)$ & $60.0 \%(9)$ \\
\hline
\end{tabular}

Data are \% (n).

AHA, anti-hyperglycemic agent.

on previous work by Gummesson $e t a l,{ }^{20}$ one would expect for every $1 \mathrm{~kg}$ of mean weight loss there is a corresponding decrease of 0.1 percentage unit in HbAlc. Therefore, in this work with a mean weight loss of $10.8 \mathrm{~kg}$, we would expect an approximate $1.1 \%$ unit decrease in HbAlc. However, a 2.3\% unit decrease in HbA1c was observed suggesting some weight-independent effect on HbA1c.

Concurrent with the clinical work described here, Melissas $e t a t^{21}$ published outcomes of a similar procedure in a cohort of six patients with T2DM that underwent a simple side-to-side jejuno-ileal anastomosis. Their preliminary results showed prolonged T2DM remission in three patients and partial remission in the other three patients post-operatively. Follow-up ranged from 6 to 42 months. Several differences between the study populations point to patients in the study by Melissas $e t$ al as having greater $\beta$-cell function and being earlier in T2DM progression: $17 \%$ vs $80 \%$ of patients on insulin prior to the procedure, average time since T2DM diagnosis of just over 5 years versus over 10 years, and median baseline HbA1c level of $7.6 \%(n=6$; range: $6.7 \%-8.4 \%)$ vs $9.4 \%$ ( $\mathrm{n}=15$; range: $8.1 \%-10.8 \%)$. Patients in both studies showed clinically relevant mean total weight loss of at least $10 \%$ post-procedure.

This proof-of-concept study is limited by a small study population with advanced disease, $80 \%$ of whom were insulin dependent and an average time since T2DM diagnosis of over 10 years, both of which are factors which have been shown to blunt the effect of bariatric surgery on T2DM. ${ }^{22}$ Several other limitations include lack of metabolic biomarkers, length of follow-up after the procedure, and no pre-determined standardized treatment algorithm for adjusting AHAs. Future studies of PJD should include longer follow-up to determine durability of effect and further characterization of mechanisms of action. Metabolic surgery involving resection such as RYGB and SG are recognized among the most potent interventions for T2DM available, but are not appropriate for overweight or normal weight patients and not routinely accepted by patients with obesity or referring physicians.

In conclusion, the results from these studies suggest that PJD may provide an anatomy sparing, low-risk, potentially reversible, metabolic procedure for poorly controlled T2DM, which does not impose significant alteration of a patient's lifestyle and may reduce the need for multiple medications. Further studies to confirm efficacy, safety, and durability of effect in T2DM are warranted.

Acknowledgements The authors wish to acknowledge the early study design input and feedback from Dr Muffazal Lakdawala (Centre for Obesity and Digestive Surgery, Mumbai, India), Dr Dave D’Alessio (Division of Endocrinology, Metabolism and Nutrition, Duke University, Durham, North Carolina, USA) and Dr Nestor de la Cruz-Munoz (Division of Laparoendoscopic and Bariatric Surgery, Florida International University, Miami, Florida, USA), and scientific input from Dr Kirk Ways and Dr David Polidori of Janssen R\&D USA.

Contributors MF was involved in the clinical study design, execution, data collection and interpretation, and manuscript review and final approval. KD was involved in clinical study execution, data collection and interpretation, and manuscript review and final approval. APC was involved in the pre-clinical study design, execution, data collection and interpretation of the pre-clinical work, and manuscript preparation and final approval. EJF was involved in the clinical study design, data interpretation, and manuscript review and final approval. RS was involved in data interpretation, manuscript preparation and final approval. MLS was involved in the clinical study design, statistical analysis, data interpretation, manuscript review and final approval. JRW was involved in the clinical study design, data interpretation, manuscript preparation and final approval. MH was involved in the data interpretation, manuscript review and final approval. RJS was involved in the pre-clinical study design, data collection and interpretation of the pre-clinical work, clinical study design, data interpretation, manuscript review and final approval. All authors have reviewed and approved the completed manuscript.

Funding Funding for this study has been provided by Ethicon, Inc and by RVO VFN64165 from MHCR to MH.

Competing interests MF, KD, APC, and MH have nothing to declare. RJS currently receives funding from Ethicon, Inc. EJF, RS, MLS, and JRW are employed by Ethicon, Inc.

Patient consent Informed consent was previously obtained from all study patients.

Ethics approval Ethics Committee of the OB Clinic, Prague, Czech Republic. Provenance and peer review Not commissioned; externally peer reviewed. Data sharing statement This manuscript includes all the data available.

Open Access This is an Open Access article distributed in accordance with the Creative Commons Attribution Non Commercial (CC BY-NC 4.0) license, which permits others to distribute, remix, adapt, build upon this work non-commercially, and license their derivative works on different terms, provided the original work is properly cited and the use is non-commercial. See: http://creativecommons.org/ licenses/by-nc/4.0/

(c) Article author(s) (or their employer(s) unless otherwise stated in the text of the article) 2017. All rights reserved. No commercial use is permitted unless otherwise expressly granted.

\section{REFERENCES}

1. Global Report on Diabetes. World Health Organization. http://apps. who.int/iris/bitstream/10665/204871/1/9789241565257_eng.pdf? ua $=1 \& u a=1$. (accessed Mar 2017).

2. Global Health Estimates 2015. Deaths by Cause, Age, Sex, by Country and by Region, 2000-2015. Geneva: World Health Organization, 2016. http://www.who.int/healthinfo/global_burden disease/GlobalCOD method_2000 2015.pdf?ua=1 (accessed Mar 2017).

3. Schauer PR, Bhatt DL, Kirwan JP, et al. Bariatric surgery versus intensive medical therapy for diabetes-3-year outcomes. $N$ Engl $J$ Med 2014;370:2002-13.

4. Rubino F, Nathan DM, Eckel RH, et al. Delegates of the $2^{\text {nd }}$ Diabetes Surgery Summit. Metabolic surgery in the treatment algorithm for Type 2 diabetes: A joint statement by International Diabetes Organizations. Diabetes Care 2016;39:861-77.

5. Stark Casagrande S, Fradkin JE, Saydah SH, et al. The prevalence of meeting $\mathrm{A} 1 \mathrm{C}$, blood pressure, and LDL goals among people with diabetes, 1988-2010. Diabetes Care 2013;36:2271-9.

6. Schauer PR, Bhatt DL, Kirwan JP, et al. Bariatric surgery versus intensive medical therapy for diabetes - 5-Year outcomes. $N$ Engl $J$ Med 2017;376:641-51. 
7. Rubino F, Schauer PR, Kaplan LM, et al. Metabolic surgery to treat type 2 diabetes: clinical outcomes and mechanisms of action. Annu Rev Med 2010;61:393-411.

8. Singh D, Laya AS, Clarkston WK, et al. Jejunoileal bypass: a surgery of the past and a review of its complications. World $\mathrm{J}$ Gastroenterol 2009;15:2277-9.

9. Baker MT. The history and evolution of bariatric surgical procedures. Surg Clin North Am 2011;91:1181-201.

10. Sánchez-Pernaute A, Rubio MÁ, Pérez Aguirre E, et al. Singleanastomosis duodenoileal bypass with sleeve gastrectomy: metabolic improvement and weight loss in first 100 patients. Surg Obes Relat Dis 2013;9:731-5.

11. Melissas J, Peppe A, Askoxilakis J, et al. Sleeve gastrectomy plus side-to-side jejunoileal anastomosis for the treatment of morbid obesity and metabolic diseases: a promising operation. Obes Surg 2012;22:1104-9.

12. Woods SC, Seeley RJ, Rushing PA, et al. A controlled high-fat diet induces an obese syndrome in rats. J Nutr 2003;133:1081-7.

13. Jandacek RJ, Heubi JE, Tso P. A novel, noninvasive method for the measurement of intestinal fat absorption. Gastroenterology 2004;127:139-44.

14. Chambers AP, Jessen L, Ryan KK, et al. Weight-independent changes in blood glucose homeostasis after gastric bypass or vertical sleeve gastrectomy in rats. Gastroenterology 2011;141:950-8.
15. Matsuda M, DeFronzo RA. Insulin sensitivity indices obtained from oral glucose tolerance testing: comparison with the euglycemic insulin clamp. Diabetes Care 1999;22:1462-70.

16. Herzberg-Schäfer SA, Staiger $\mathrm{H}$, Heni M, et al. Evaluation of fasting state-/oral glucose tolerance test-derived measures of insulin release for the detection of genetically impaired $\beta$-cell function. PLoS One 2010;5:e14194.

17. Dindo D, Demartines N, Clavien PA. Classification of surgical complications: a new proposal with evaluation in a cohort of 6336 patients and results of a survey. Ann Surg 2004;240:205-13.

18. Stemmer K, Bielohuby M, Grayson BE, et al. Roux-en-Y gastric bypass surgery but not vertical sleeve gastrectomy decreases bone mass in male rats. Endocrinology 2013;154:2015-24.

19. Aminian A, Brethauer SA, Kirwan JP, et al. How safe is metabolic/ diabetes surgery? Diabetes Obes Metab 2015;17:198-201.

20. Gummesson A, Nyman E, Knutsson M, et al. Effect of weight reduction on glycated haemoglobin in weight loss trials in patients with type 2 diabetes. Diabetes Obes Metab 2017:1-11.

21. Melissas J, ErenTaskin H, Peirasmakis D, et al. A Simple fooddiverting operation for Type 2 diabetes treatment. Preliminary results in humans with BMI $28-32 \mathrm{~kg} / \mathrm{m}(2)$. Obes Surg 2017;27:22-9.

22. Dixon JB, Zimmet P, Alberti KG, et al. Bariatric surgery: an IDF statement for obese Type 2 diabetes. Diabet Med 2011;28:628-42. 